\title{
COEFFICIENTS FOR THE STUDY OF RUNGE-KUTTA INTEGRATION PROCESSES
}

\author{
J. C. BUTCHER
}

(received 21 May 1962)

\section{Introduction}

We consider a set of $n$ first order simultaneous differential equations in the dependent variables $y_{1}, y_{2}, \cdots, y_{n}$ and the independent variable $x$

(1)

$$
\begin{gathered}
\frac{\mathrm{d} y_{1}}{\mathrm{~d} x}=f_{1}\left(y_{1}, y_{2}, \cdots, y_{n}\right), \\
\frac{\mathrm{d} y_{2}}{\mathrm{~d} x}=f_{2}\left(y_{1}, y_{2}, \cdots, y_{n}\right), \\
\vdots \\
\frac{\mathrm{d} y_{n}}{\mathrm{~d} x}=f_{n}\left(y_{1}, y_{2}, \cdots, y_{n}\right) .
\end{gathered}
$$

No loss of generality results from taking the functions $f_{1}, f_{2}, \cdots, f_{n}$ to be independent of $x$, for if this were not so an additional dependent variable $y_{n+1}$, can be introduced which always equals $x$ and thus satisfies the differential equation

$$
\frac{\mathrm{d} y_{n+1}}{\mathrm{~d} x}=1
$$

When convenient we will write the set of equations (1) in one of the vector forms

$$
\frac{\mathrm{d} y_{i}}{\mathrm{~d} x}=f_{i}(y), \quad(i=1,2, \cdots, n),
$$

or

$$
\frac{\mathrm{d} y}{\mathrm{~d} x}=f(y)
$$

We will suppose that the values $y=y_{0}$ are given at $x=x_{0}$ and that it is required to find an approximation $\hat{y}$ to the value of $y$ at the point $x=x_{0}+h$.

A Runge-Kutta process is a solution to this problem defined by the equations 


$$
\begin{gathered}
g^{(I)}=f\left(y_{0}+h \sum_{J=1}^{\nu} a_{I J} g^{(J)}\right), \\
\hat{y}=y_{0}+h \sum_{I=1}^{\nu} b_{I} g^{(I)},
\end{gathered}
$$

where $\boldsymbol{g}^{(I)}(I=1,2, \cdots, v)$ is a set of $n$ element vectors and the numbers $a_{I J}, b_{I}$ are parameters which distinguish different processes of this type. If the vectors $g^{(1)}, g^{(2)}, \cdots, g^{(v)}$ are to be evaluated one at a time in this order then the parameters $a_{I J}$ must satisfy the conditions

$$
a_{I J}=0, \quad(J \geqq I) .
$$

However, since implicit processes are also possible we do not impose this restriction.

The simplest examples of Runge-Kutta processes are, for $v=2$, that due to Runge [1] and for $v=3$, that due to Kutta [2]. The non-vanishing $a_{I J}, b_{I}$ are, for the Runge process

$$
a_{21}=\frac{1}{2}, \quad b_{2}=1,
$$

and for the Kutta process

$$
\begin{array}{ll}
a_{21}=\frac{1}{2}, & \\
a_{31}=-1, & a_{32}=2, \\
b_{1}=b_{3}=\frac{1}{6}, & b_{2}=\frac{2}{3} .
\end{array}
$$

The values of these quantities are chosen so that the power series expansion for $\hat{y}$ defined by (4) and (5) should be identical with that for $\boldsymbol{y}$ up to the terms in $h^{2}$ (in the Runge case) or $h^{3}$ (in the Kutta case). Other processes of higher order are due to Kutta [2], Nyström [3] and Gill [4].

It is the purpose of the present paper to derive expressions for the various terms of the expansions for $\boldsymbol{y}$ and $\hat{y}$ and to present tables of certain coefficients which allow these terms to be written down immediately.

Thus the scope of the paper is similar to that of Merson [5] who used an operational method to study these expansions. Certain vectors occurring in the expansion, which are called "elementary differentials" in the present paper were shown to bear a $(1-1)$ correspondence to the rooted trees of topology and a calculus was developed for manipulating them and thus deriving the terms of the expansions in given cases.

\section{The Taylor expansion for $y$}

It is convenient to define from the functions $f(y)$ occurring in (3), a set of $n$ element vectors which we will call elementary differentials of given order and degree. The order is an integer greater than zero while the degree is an 
integer less than the order and greater than zero. If the order is unity the degree is not defined.

$f=f(y)$ is the only elementary differential of order 1 .

$F$ is an elementary differential of order $r$ and degree $s$

if

$$
\boldsymbol{F}=\sum_{j_{1}=1}^{n} \sum_{j_{2}=1}^{n} \cdots \sum_{j_{t}=1}^{n} \frac{\partial^{s} f}{\partial y_{j_{1}} \partial y_{j_{2}} \cdots \partial y_{j_{d}}} F_{1 s_{1}} F_{2 s_{1}} \cdots F_{8 j_{q}},
$$

where $F_{i j}$ is element number $j$ of an elementary differential $F_{i}$ of order $r_{i}(i=1,2, \cdots, s)$ such that

$$
r=1+r_{1}+r_{2}+\cdots+r_{s} .
$$

For simplicity the elementary differential (7) will be written as

$$
F=\left\{F_{1} F_{2} \cdots F_{2}\right\} \text {. }
$$

In general $\boldsymbol{F}_{1}, \boldsymbol{F}_{2} \cdots \boldsymbol{F}_{8}$ need not be distinct. Let us suppose for example that $\boldsymbol{F}_{1}, \boldsymbol{F}_{2}, \cdots \boldsymbol{F}_{\sigma}$ are distinct but each of $\boldsymbol{F}_{\sigma+1}, \cdots \boldsymbol{F}_{8}$ is identical to one of them so that in all $F_{i}(i=1,2, \cdots \sigma)$ occurs $\mu_{i}$ times amongst $F_{1}, F_{2}$, $\cdots F_{\text {a }}$. In this case we have

$$
\begin{aligned}
& r=1+\mu_{1} r_{1}+\mu_{2} r_{2}+\cdots+\mu_{\sigma} r_{\sigma}, \\
& s=\mu_{1}+\mu_{2}+\cdots+\mu_{\sigma}
\end{aligned}
$$

and we write $F$ in (8) as

$$
F=\left\{F_{1}^{\mu_{1}} F_{2}^{\mu_{2}} \cdots F_{\sigma}^{\mu_{\sigma}}\right\} .
$$

A further convenient abbreviation of the notation is possible for an elementary differential of degree 1 . For example $\{\boldsymbol{F}\}$ where $\boldsymbol{F}$ is given by (9) will be written as

$$
\left\{{ }_{2} F_{1}^{\mu_{1}} F_{2}^{\mu_{2}} \cdots F_{\sigma}^{\mu_{\sigma}}\right\}_{2}
$$

and generally $\left\{_{a-1} F\right\}_{a-1}$ will be written as

$$
\left\{F_{1}^{\mu_{1}} F_{2}^{\mu_{2}} \cdots F_{\sigma}^{\mu_{\sigma}}\right\}_{a},
$$

for any positive integer $a$.

If $\boldsymbol{F}_{1}, \boldsymbol{F}_{2}, \cdots \boldsymbol{F}_{s}$ in $(7)$ are linear combinations of elementary differentials then $\boldsymbol{F}$ will also be a linear combination of elementary differentials. However, the notations (8) and (9) will still be used in these cases and it is easy to see for example that

$$
\begin{aligned}
\left\{a\left(b F_{1}^{\prime}+c F_{1}^{\prime \prime}\right) F_{2} \cdots F_{s}\right\}_{a}= & b\left\{_{a} F_{1}^{\prime} F_{2} \cdots F_{s}\right\}_{a} \\
& \left.+c{ }_{a} F_{1}^{\prime \prime} F_{2} \cdots F_{s}\right\}_{a}
\end{aligned}
$$

for constants $b$ and $c$. 
It is convenient to adopt one final convention. The symbol $\{1\}$ will be identified with $\boldsymbol{f}$.

The importance of the elementary differentials results from the following theorems.

THEOREM 1. The differential coefficient (with respect to $x$ ) of any elementary differential of order $r$ is a linear combination with non-negative integral coefficients of the elementary differentials of order $r+1$.

The proof is by induction. For $r=1, f$ is the only elementary differential and

$$
\frac{\mathrm{d}}{\mathrm{d} x} \boldsymbol{f}(\boldsymbol{y})=\sum_{i=1}^{n} \frac{\partial f}{\partial y_{i}} \frac{\mathrm{d} y_{i}}{\mathrm{~d} x}=\{\boldsymbol{f}\}
$$

an elementary differential of order 2 (in fact the only one of this order). If the operator $D$ replaces an elementary differential on which it operates by its derivative we have for the derivative of the elementary differential (7)

$$
\begin{aligned}
\mathrm{D} F=\left\{f F_{1} F_{2} \cdots F_{s}\right\}+\left\{\left(\mathrm{D} F_{1}\right) F_{2} \cdots F_{s}\right\} & +\left\{F_{1}\left(\mathrm{D} F_{2}\right) \cdots F_{s}\right\} \\
& \cdots+\left\{F_{1} F_{2} \cdots\left(\mathrm{D} F_{s}\right)\right\}
\end{aligned}
$$

each term of which, by the induction hypothesis, is of the correct form.

The following corollaries are easily proved by induction.

COROLLARY 1. The sum of the coefficients of the elementary differentials in $\mathrm{DF}$ is $r$.

COROLlARY 2. $\mathrm{D}^{t} F$ is a linear combination with non negative integral coefficients of the elementary differentials of order $r+t$ and the sum of the coefficients is $(r+t-1) ! /(r-1)$ !

For the case of $D^{t} f$ we see that each term is of order $t+1$ and that the sum of the coefficients is $t$ ! For this case we prove the stronger theorem.

THEOREM 2. When $D^{t} f$ is written as a linear combination of the elementary differentials of order $t+1$ none of the coefficients vanishes.

For $t=1$ we have

$$
\mathrm{D} \boldsymbol{f}=\{\boldsymbol{f}\}
$$

and this is clearly the only elementary differential of order 2. Let us assume the result for $t<r-1$ and consider the elementary differential (8). Two cases arise

(i) $\boldsymbol{F}=\left\{\boldsymbol{f}^{\prime-1}\right\}$,

(ii) one at least of $\boldsymbol{F}_{1}, \boldsymbol{F}_{2} \cdots \boldsymbol{F}_{3}$ is not $\boldsymbol{f}$. Suppose for example $\boldsymbol{F}_{1}$ is of order $r_{1}$ where $1<r_{1}<r$.

In case (i) $\boldsymbol{F}$ occurs in $\mathrm{D}\left\{\boldsymbol{f}^{\boldsymbol{r}-2}\right\}$ and by hypothesis $\left\{\boldsymbol{f}^{r-2}\right\}$ occurs in $\mathrm{D}^{r-2} \boldsymbol{f}$.

In case (ii) $\boldsymbol{F}$ occurs in $D F^{\prime}$ where 


$$
F^{\prime}=\left\{\left(\mathrm{D}^{r_{1}-1} f\right) F_{2} F_{3} \cdots F_{s}\right\}
$$

and $F^{\prime}$ occurs in $D^{r-2} f$.

By repeated application of (10) we can write down the terms of $D^{t} F$ or in particular of $\mathrm{D}^{\boldsymbol{t}} \boldsymbol{f}$. We find for example

$$
\begin{aligned}
& \mathrm{D} \boldsymbol{f}=\{\boldsymbol{f}\}, \\
& \mathrm{D}^{2} \boldsymbol{f}=\left\{{ }_{2} \boldsymbol{f}\right\}_{2}+\left\{\boldsymbol{f}^{2}\right\}, \\
& \left.\mathrm{D}^{3} \boldsymbol{f}=\left\{{ }_{3} \boldsymbol{f}\right\}_{3}+3\{\boldsymbol{f}\{\boldsymbol{f}\}\}+{ }_{2} \boldsymbol{f}^{2}\right\}_{2}+\left\{\boldsymbol{f}^{3}\right\} .
\end{aligned}
$$

The Taylor expansion for $\boldsymbol{y}$ we find by evaluating these expressions at $\boldsymbol{y}=\boldsymbol{y}_{\mathbf{0}}$. If evaluation at this point is understood for all elementary differentials occurring in such a context as this we may write

$$
\begin{aligned}
y & =y_{0}+\sum_{r=1}^{\infty} \frac{h^{r}}{r !} \mathrm{D}^{r-1} f \\
& =y_{0}+\sum_{i=1}^{\infty} \frac{h^{(i)}}{r^{(i) !}} \alpha^{(i)} F^{(i)}
\end{aligned}
$$

where $F^{(1)}(=f), F^{(2)}, F^{(3)}, \cdots$ are the complete set of elementary differentials with orders $r^{(1)}(=1), r^{(2)}, r^{(3)}, \cdots$ in non decreasing order and $\alpha^{(i)}$ the coefficient with which $F^{(i)}$ occurs in $D^{r^{(i)}-1} f$.

The first few terms of (11) are easily found

$$
\begin{aligned}
y=y_{0}+h \boldsymbol{f} & +\frac{h^{2}}{2 !}\{f\}+\frac{h^{3}}{3 !}\left(\left\{_{2} f\right\}_{2}+\left\{f^{2}\right\}\right) \\
& +\frac{h^{4}}{4 !}\left(\left\{{ }_{3} f\right\}_{3}+3\{f\{f\}\}+\left\{f_{2}\right\}_{2}+\left\{f^{3}\right\}\right)+\cdots
\end{aligned}
$$

For elementary differentials of high order the use of $(10)$ to find the coefficients $\alpha^{(i)}$ becomes increasingly tedious. However, a simpler method is to use the following theorem.

THEOREM 3. If $\left(h^{3} / r !\right)$ a is the coefficient of $\boldsymbol{F}$, defined by (9) in the expansion (11) and $F_{1}, F_{2}, \cdots F_{\sigma}$ are all distinct then

$$
\alpha=(r-1) ! \prod_{i=1}^{\sigma} \frac{1}{\mu_{i} !}\left(\frac{\alpha_{i}}{r_{i} !}\right)^{\mu_{i}}
$$

where $\left(h^{r_{i}} / r_{i} !\right) \alpha_{i}(i=1,2, \cdots \sigma)$ is the coefficient of $F_{i}$ in (11).

To prove this result we substitute the expansion (11) into (3) and compare terms.

The coefficient of $\boldsymbol{F}$ on the left hand side is

$$
\frac{\alpha h^{r-1}}{(r-1) !}
$$


while the right hand side is given by the Taylor expansion

$$
\boldsymbol{f}+\sum_{s=1}^{\infty} \sum_{j_{1}=1}^{n} \sum_{s_{1}=1}^{n} \cdots \sum_{j_{s}=1}^{n} \frac{1}{s !} \frac{\partial^{*} f}{\partial y_{j_{1}} \partial y_{s_{2}} \cdots \partial y_{s_{s}}}\left(\eta_{s_{1}} \eta_{s_{2}} \cdots \eta_{s_{s}}\right)
$$

where

$$
\eta=y-y_{0}=\sum_{i=1}^{\infty} \frac{h^{(i)}}{r^{(i) !}} \alpha^{(i)} F^{(i)}
$$

Thus the right hand side may be written as

$$
f+\sum_{s=1}^{\infty} \frac{1}{s !}\left\{\eta^{s}\right\}
$$

or, adopting the conventions stated, as

$$
\left\{1+\sum_{s=1}^{\infty} \frac{\eta^{s}}{s !}\right\}=\left\{e^{n}\right\}
$$

We see that in (14) $\boldsymbol{F}$ occurs only in $1 / s !\left\{\eta^{s}\right\}$ where $s$ is the degree of $\boldsymbol{F}$. Furthermore, the only terms of this that contribute come from

$$
\frac{1}{s !}\left\{\left(\sum_{i=1}^{\sigma} \frac{h^{r_{1}}}{r_{i} !} \alpha_{i} F_{i}\right)^{s}\right\}
$$

and the coefficient of $\boldsymbol{F}=\left\{\boldsymbol{F}_{1}^{\mu_{1}} \boldsymbol{F}_{2}^{\mu_{2}} \cdots F_{\sigma}^{\mu_{\sigma}}\right\}$ in this is

$$
\frac{h^{r-1}}{s !}\left(\begin{array}{c}
s \\
\mu_{1}, \mu_{2}, \cdots, \mu_{\sigma}
\end{array}\right) \prod_{i=1}^{\sigma}\left(\frac{\alpha_{i}}{r_{i} !}\right)^{\mu_{c}}
$$

where $\left(\begin{array}{c}s \\ \mu_{1}, \mu_{2}, \cdots, \mu_{\sigma}\end{array}\right)$ is the multinomial coefficient

$$
s ! / \prod_{i=1}^{\sigma}\left(\mu_{i} !\right)
$$

Comparing (13) with (15) we obtain the result (12).

If (12) is used to compute the coefficients $\alpha$, for the elementary differentials of order $r$ we may use as an independent check the fact that the sum of the coefficients is $(r-1)$ ! (Theorem 1, Corollary 1.)

In table 1 the elementary differentials of orders up to 8 are tabulated in the second column against order in the first column. The corresponding values of $\alpha$ are in the third column. 
TABLE 1

\begin{tabular}{|c|c|c|c|c|c|c|c|c|c|}
\hline$r$ & $F$ & $\alpha$ & $\beta$ & $\gamma$ & $r$ & $F$ & $\alpha$ & $\beta$ & $\gamma$ \\
\hline 1 & $f$ & 1 & 1 & 1 & $\begin{array}{l}7 \\
7\end{array}$ & $\left\{\begin{array}{l}\left\{_{3}\left\{f, f^{2}\right\}_{3}\right. \\
\left\{f^{4}\right\}\end{array}\right.$ & $\begin{array}{l}6 \\
1\end{array}$ & $\begin{array}{r}360 \\
30\end{array}$ & $\begin{array}{l}420 \\
210\end{array}$ \\
\hline 2 & $\{f\}$ & 1 & 1 & 2 & $\begin{array}{l}7 \\
7\end{array}$ & $\left\{\begin{array}{l}\left\{_{2}\left\{{ }_{3} f_{3} f\right\}_{2}\right. \\
\left\{_{2}\left\{f_{3} f^{2}\right\}_{f} f\right\}_{3}\end{array}\right.$ & $\begin{array}{l}5 \\
5\end{array}$ & $\begin{array}{l}720 \\
360\end{array}$ & $\begin{array}{r}1008 \\
504\end{array}$ \\
\hline 3 & $\{, f\}_{2}$ & 1 & 2 & 6 & 7 & $\left.f_{2}\{(f\} f\}\right\}_{2}$ & 15 & 720 & 336 \\
\hline 3 & $\left\{f^{2}\right\}$ & 1 & 1 & 3 & $\begin{array}{l}7 \\
7\end{array}$ & 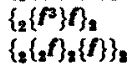 & $\begin{array}{r}5 \\
10\end{array}$ & $\begin{array}{l}120 \\
720\end{array}$ & $\begin{array}{l}168 \\
504\end{array}$ \\
\hline 4 & $\left\{{ }_{s}\right\}_{3}$ & 1 & 6 & 24 & 7 & $\left\{_{2}\left\{f^{2}\right\}(f)\right\}_{2}$ & 10 & 360 & 252 \\
\hline 4 & $\left({ }_{2} f^{2}\right)_{2}$ & 1 & $\mathbf{3}$ & 12 & 7 & $\left\{,\{, f\}_{1} I^{2}\right\}_{2}$ & 10 & 360 & 252 \\
\hline 4 & $\{\{n\}$ & 3 & 6 & 8 & 7 & $\left\{_{\Omega}\left\{f^{2}\right\} f^{2}\right\}_{2}$ & 10 & 180 & 126 \\
\hline 4 & $\{\boldsymbol{f}\}$ & 1 & 1 & 4 & 7 & $\left\{_{2}\{f\}^{2} f\right\}_{2}$ & 15 & 360 & 168 \\
\hline 5 & $\{, f\}_{a}$ & 1 & 24 & 120 & 7 & $\begin{array}{l}\left\{_{2}\{l\} I^{0}\right\}_{2} \\
\left\{f^{f}\right\}_{2}\end{array}$ & $\begin{array}{r}10 \\
1\end{array}$ & $\begin{array}{r}120 \\
6\end{array}$ & $\begin{array}{l}84 \\
42\end{array}$ \\
\hline 5 & $\left\{f_{3}\right\}_{3}$ & 1 & 12 & 60 & 7 & $\{\{, f\}, f\}$ & 6 & 720 & 840 \\
\hline 5 & $\left\{_{3}\{f \mid f\}_{2}\right.$ & 3 & 24 & 40 & 7 & $\left\{\left\{_{3} f^{2}\right\}_{3} f\right\}$ & 6 & 360 & 420 \\
\hline 5 & $\left\{g^{\prime}\right\}_{2}$ & 1 & 4 & 20 & 7 & $\left\{\left\{_{2}\{f\} f\right\}_{2} f\right\}$ & 18 & 720 & 280 \\
\hline 5 & $\left\{\left\{_{2} f\right\}_{2} \cap\right\}$ & 4 & 24 & 30 & 7 & $\left\{\left\{, f^{3}\right\}, f\right\}$ & 6 & 120 & 140 \\
\hline 5 & $\{(\boldsymbol{r}\} \boldsymbol{f}\}$ & 4 & 12 & 15 & 7 & $\left\{\left\{\left\{_{2} f\right\}_{2} f\right\} f\right\}$ & 24 & 720 & 210 \\
\hline 5 & $\left\{\left(\boldsymbol{f}^{*}\right\}\right.$ & 3 & 12 & 20 & 7 & $\left\{\left\{\left\{f^{2}\right\} f\right\} f\right\}$ & 24 & 360 & 105 \\
\hline 5 & $\{\{\boldsymbol{n} \boldsymbol{\Gamma}\}$ & 6 & 12 & 10 & 7 & $\left\{\left\{\{\boldsymbol{f}\}^{2}\right\} \boldsymbol{f}\right\}$ & 18 & 360 & 140 \\
\hline \multirow[t]{2}{*}{5} & $\{\boldsymbol{f}\}$ & 1 & 1 & 5 & 7 & $\left\{\left\{\{f\} f^{2}\right\} f\right\}$ & 36 & 360 & 70 \\
\hline & & & & & 7 & $\left\{\left\{\mathbf{f}^{\prime}\right\} \boldsymbol{f}\right\}$ & 6 & 30 & 35 \\
\hline 6 & $\left\{f_{6} f\right\}_{6}$ & 1 & 120 & 720 & 7 & $\left\{\left\{_{3} f\right\}_{3}\{f\}\right\}$ & 15 & 720 & 336 \\
\hline 6 & $\left\{f^{n}\right\}_{4}$ & 1 & 60 & 360 & 7 & $\left\{\left\{_{2} \boldsymbol{r}^{2}\right\}_{3}\{f\}\right\}$ & 15 & 360 & 168 \\
\hline 6 & $\{(f) t\}$ & 3 & 120 & 240 & 7 & $\{\{\{\boldsymbol{f}\} \boldsymbol{f}\}\{\boldsymbol{f}\}\}$ & 45 & 720 & 112 \\
\hline 6 & $\left\{x^{2}\right\}_{2}$ & 1 & 20 & 120 & 7 & $\left\{\left\{\mathbf{f}^{3}\right\}\{\boldsymbol{f}\}\right\}$ & 15 & 120 & 56 \\
\hline 6 & $\left\{,\{, f\}_{2} f\right\}_{2}$ & 4 & 120 & 180 & 7 & $\left\{\left\{{ }_{3} f\right\}_{9} f^{2}\right\}$ & 15 & 360 & 168 \\
\hline 6 & $\left\{_{3}\left\{f^{2}\right) f\right\}_{3}$ & 4 & 60 & 90 & 7 & $\left\{\left\{f_{2}\right\}_{2} f_{2}{ }^{2}\right\}$ & 15 & 180 & 84 \\
\hline 6 & $\left\{_{1}(f)^{2}\right\}_{2}$ & 3 & 60 & 120 & 7 & $\left\{\{\{f\} f\} f^{2}\right\}$ & 45 & 360 & 56 \\
\hline 6 & 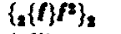 & 6 & 60 & 60 & 7 & $\left\{\left\{f^{3}\right\} \boldsymbol{F}^{\mathbf{2}}\right\}$ & 15 & 60 & 28 \\
\hline 6 & $\left\{x_{2} \boldsymbol{F}^{*}\right\}_{\mathbf{z}}$ & 1 & 5 & 30 & 7 & $\left\{\left\{_{2} f\right\}_{2}{ }^{2}\right\}$ & 10 & 360 & 252 \\
\hline 6 & \{\}$\left.\left._{3} f,\right\}_{2} f\right\}$ & 5 & 120 & 144 & 7 & $\left\{\left\{_{2} f_{2}\left\{f^{2}\right\}\right\}\right.$ & 20 & 360 & 126 \\
\hline 6 & $\left.\left\{l_{2} \boldsymbol{I}^{2}\right\}_{\mathrm{g}} \boldsymbol{f}\right\}$ & 5 & 60 & 72 & 7 & $\left\{\left\{\boldsymbol{f}^{2}\right\}^{2}\right\}$ & 10 & 90 & 63 \\
\hline 6 & $\{(\{\boldsymbol{\gamma}\}) \boldsymbol{f}\}$ & 15 & 120 & 48 & 7 & $\left\{\left\{_{2} f\right\}_{2}\{f\} f\right\}$ & 60 & 720 & 84 \\
\hline B & $\left\{\left\{\boldsymbol{P}^{\prime}\right\}\right)$ & 5 & 20 & 24 & 7 & $\left\{\left\{f^{2}\right\}\{f\} f\right\}$ & 60 & 360 & 42 \\
\hline 6 & $\left\{\left\{_{2} f\right\}_{2}\{f\}\right\}$ & 10 & 120 & 72 & 7 & $\left\{\left\{_{2} f\right\}_{2} P\right\}$ & 20 & 120 & 42 \\
\hline 6 & $\left\{\left\{f^{2}\right\}\{f\}\right\}$ & 10 & 60 & 36 & 7 & $\left\{\left\{\boldsymbol{f}^{2}\right\} \mathbf{f}^{\mathbf{3}}\right\}$ & 20 & 60 & 21 \\
\hline 6 & $\left\{\left\{, f, f_{2}^{2}\right\}\right.$ & 10 & 60 & 36 & 7 & $\left\{\{\boldsymbol{f}\}^{3}\right\}$ & 15 & 120 & 56 \\
\hline 6 & $\left\{(\boldsymbol{r}\}^{\boldsymbol{r}}\right\}$ & 10 & 30 & 18 & 7 & $\left\{\{f\}^{2} f^{2}\right\}$ & 45 & 180 & 28 \\
\hline 6 & $\left\{\{\boldsymbol{f}\}^{2} \boldsymbol{f}\right\}$ & 15 & 60 & 24 & 7 & $\left\{\{\boldsymbol{f}\} \boldsymbol{f}^{4}\right\}$ & 15 & 30 & 14 \\
\hline 6 & $\left\{\{\boldsymbol{f}\} \mathbf{r}^{\mathbf{2}}\right\}$ & 10 & 20 & 12 & 7 & $\left\{\boldsymbol{f}^{\mathbf{s}}\right\}$ & 1 & 1 & 7 \\
\hline \multirow[t]{2}{*}{6} & $\left\{f^{s}\right\}$ & 1 & 1 & 6 & & & & & \\
\hline & & & & & 8 & $\left\{f_{7}\right\}_{2}$ & 1 & 5040 & 40320 \\
\hline 7 & $\{,\}_{6}$ & l & 720 & 5040 & 8 & $\left\{f^{2}\right\}_{6}$ & 1 & 2520 & 20160 \\
\hline 7 & $\left\{f^{2}\right\}_{6}$ & 1 & 360 & 2520 & 8 & $\left\{_{5}(f) f\right\}_{5}$ & 3 & 5040 & 13440 \\
\hline 7 & $\{(f) f\}_{a}$ & 3 & 720 & 1680 & 8 & $\left\{{ }_{5} f\right\}_{s}$ & 1 & 840 & 6720 \\
\hline 7 & $\left\{f^{3}\right\}_{4}$ & 1 & 120 & 840 & 8 & $\left.{ }_{4}\left\{{ }_{2} f\right\}_{8} f\right\}_{4}$ & 4 & 5040 & 10080 \\
\hline 7 & $\left.\left\{x_{2}\right\}_{2}\right\}_{2} f_{3}$ & 4 & 720 & 1260 & 8 & 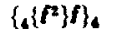 & 4 & 2520 & 5040 \\
\hline 7 & $\left\{g\left(f^{2}\right\} f\right\}_{3}$ & 4 & 360 & 630 & 8 & $\left\{_{d}\{\boldsymbol{f}\}^{2}\right\}_{4}$ & 3 & 2520 & 6720 \\
\hline 7 & $\left.\hat{f}_{3}\{f\}^{2}\right\}_{3}$ & $\mathbf{3}$ & $\mathbf{3 6 0}$ & $\mathbf{8 4 0}$ & 8 & $\left\{{ }_{A}\{f\} f^{2}\right\}_{4}$ & 6 & 2520 & 3360 \\
\hline
\end{tabular}


TABLE 1 (continued)

\begin{tabular}{|c|c|c|c|c|c|c|c|c|c|}
\hline$r$ & $\boldsymbol{F}$ & $\alpha$ & $\beta$ & $\gamma$ & $r$ & $F$ & $\alpha$ & $\beta$ & $\gamma$ \\
\hline 8 & $\left\{{ }_{4} f^{4}\right\}_{4}$ & 1 & 210 & 1680 & 8 & $\left\{\left\{\left\{f_{2} f^{2}\right\}_{2} f\right\} f\right\}$ & 35 & 2520 & 576 \\
\hline 8 & $\left\{_{3}\left\{{ }_{3} f\right\}_{3} f\right\}_{3}$ & 5 & 5040 & 8064 & 8 & $\{(\{\{f\} f) f\}\}$ & 105 & 5040 & 384 \\
\hline 8 & $\left.\left\{{ }_{3}\left\{z_{2}\right\}_{2}\right\}_{2} f\right\}_{3}$ & 5 & 2520 & 4032 & 8 & $\left.\left\{\left\{f^{3}\right\} f\right\} f\right\}$ & 35 & 840 & 192 \\
\hline 8 & $\left\{_{3}\{(f) f\} f\right\}_{3}$ & 15 & 5040 & 2688 & 8 & $\left\{\left\{\left\{_{2} f_{2}\{f\}\right\} f\right)\right.$ & 70 & 5040 & 576 \\
\hline 8 & $\left.{ }_{3}\left\{f^{3}\right\} f\right\}_{3}$ & 5 & 840 & 1344 & 8 & $\left\{\left\{\left\{f^{2}\right\}\{f\}\right\} f\right\}$ & 70 & 2520 & 288 \\
\hline 8 & $\left\{_{3}\left\{{ }_{2} f\right\}_{2}\{f\}\right\}_{3}$ & 10 & 5040 & 4032 & 8 & $\left\{\left\{\left\{\left\{_{2} f\right\}_{2} f^{2}\right\} f\right\}\right.$ & 70 & 2520 & 288 \\
\hline 8 & $\left\{_{3}\left\{f^{2}\right\}\{f\}\right\}_{3}$ & 10 & 2520 & 2016 & 8 & $\left\{\left\{\left\{\boldsymbol{f}^{2}\right\} \boldsymbol{f}^{2}\right\} \boldsymbol{f}\right\}$ & 70 & 1260 & 144 \\
\hline 8 & $\left\{{ }_{3}\left\{{ }_{2} f\right\}_{2} f^{2}\right\}_{3}$ & 10 & 2520 & 2016 & 8 & $\left\{\left\{\{f\}^{2} f\right\} f\right\}$ & 105 & 2520 & 192 \\
\hline 8 & $\left\{_{3}\left\{f^{2}\right\} f^{2}\right\}_{3}$ & 10 & 1260 & 1008 & 8 & $\left\{\left\{\{f\} \boldsymbol{F}^{3}\right\} \cap\right\}$ & 70 & 840 & 96 \\
\hline 8 & $\left\{_{3}\{f\}^{2} f\right\}_{3}$ & 15 & 2520 & 1344 & 8 & $\{\{\boldsymbol{F}\} \boldsymbol{f}\}$ & 7 & 42 & 48 \\
\hline 8 & $\left\{3\{f\} f^{3}\right\}_{3}$ & 10 & 840 & 672 & 8 & $\left\{\{\boldsymbol{f} f\}_{4}\{f\}\right\}$ & 21 & 5040 & 1920 \\
\hline 8 & $\left\{{ }_{3} f^{5}\right\}_{3}$ & 1 & 42 & 336 & 8 & $\left\{\left\{_{3} f^{2}\right\}_{3}\{f\}\right\}$ & 21 & 2520 & 960 \\
\hline 8 & $\left\{{ }_{2}\left\{f_{4} f\right\}_{4} f\right\}_{\mathrm{g}}$ & 6 & 5040 & 6720 & 8 & $\left\{\left\{_{2}\{f\} f\right\}_{2}\{f\}\right\}$ & 63 & 5040 & 640 \\
\hline 8 & $\left\{\left\{_{2}\left\{{ }_{3} f^{2}\right\}_{3} f\right\}_{2}\right.$ & 6 & 2520 & 3360 & 8 & $\left.\left\{{ }_{2} f^{3}\right\}_{2}\{f\}\right\}$ & 21 & 840 & 320 \\
\hline 8 & $\left\{_{2}\left\{_{2}\{f\} f\right\}_{2} f\right\}_{2}$ & 18 & 5040 & 2240 & 8 & $\left\{\left\{\left\{{ }_{2} f\right\}_{2} f\right\}\{f\}\right\}$ & 84 & 5040 & 480 \\
\hline 8 & $\left\{{ }_{2}\left\{2^{f^{3}}\right\}_{2} f\right\}_{2}$ & 6 & 840 & 1120 & 8 & $\left\{\left\{\left\{f^{2}\right\} \boldsymbol{f}\right\}\{f\}\right\}$ & 84 & 2520 & 240 \\
\hline 8 & 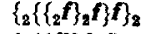 & 24 & 5040 & 1680 & 8 & $\left\{\left\{\{\boldsymbol{f}\}^{2}\right\}\{\boldsymbol{f}\}\right\}$ & 63 & 2520 & 320 \\
\hline 8 & $\left\{_{2}\left\{\left\{f^{2}\right\} f\right\} f\right\}_{2}$ & 24 & 2520 & 840 & 8 & $\left\{\left\{\{f\} f^{2}\right\}\{f\}\right\}$ & 126 & 2520 & 160 \\
\hline 8 & $\left\{_{2}\left\{\{f\}^{2}\right\}\right\}_{2}$ & 18 & 2520 & 1120 & 8 & $\left\{\left\{\boldsymbol{f}^{4}\right\}\{\boldsymbol{I}\}\right\}$ & 21 & 210 & 80 \\
\hline 8 & $\left\{_{2}\left\{\{f\} f^{2}\right\} f\right\}_{2}$ & 36 & 2520 & 560 & 8 & $\left.\left\{{ }_{4}{ }\right\}_{4} f^{2}\right\}$ & 21 & 2520 & 960 \\
\hline 8 & $\left\{{ }_{2}\left\{f^{t}\right\} f\right\}_{2}$ & 6 & 210 & 280 & 8 & $\left\{\left\{_{3} \boldsymbol{F}^{\mathbf{2}}\right\}_{3} \boldsymbol{f}^{\mathbf{2}}\right\}$ & 21 & 1260 & 480 \\
\hline 8 & 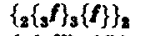 & 15 & 5040 & 2688 & 8 & $\left\{\left\{_{9}\{f) f\right\}_{2} f^{2}\right\}$ & 63 & 2520 & 320 \\
\hline 8 & $\left\{_{2}\left\{_{2} f^{2}\right\}_{2}\{f\}\right\}_{2}$ & 15 & 2520 & 1344 & 8 & $\left\{\left\{_{2} f^{3}\right\}_{2} f^{2}\right\}$ & 21 & 420 & 160 \\
\hline 8 & $\left\{_{2}\left\{\{\boldsymbol{I} \boldsymbol{f}\{\boldsymbol{f}\}\}_{2}\right.\right.$ & 45 & 5040 & 896 & 8 & $\left\{\left\{\left\{_{2} f\right\}_{2} f\right\} f^{2}\right\}$ & 84 & 2520 & 240 \\
\hline 8 & $\left.{ }_{2}\left\{\boldsymbol{f}^{3}\right\}\{\boldsymbol{f}\}\right\}_{2}$ & 15 & 840 & 448 & 8 & $\left\{\left\{\left\{f^{2}\right\} f\right\} f^{2}\right\}$ & 84 & 1260 & 120 \\
\hline 8 & $\left\{_{2}\left\{{ }_{3} f_{3} f^{2}\right\}_{2}\right.$ & 15 & 2520 & 1344 & 8 & $\left\{\left\{\{f\}^{2}\right\} f^{2}\right\}$ & 63 & 1260 & 160 \\
\hline 8 & $\left\{_{2}\left\{_{2} f^{2}\right\}_{2} f^{2}\right\}_{2}$ & 15 & 1260 & 672 & 8 & $\left\{\left\{\{\boldsymbol{f}\} \boldsymbol{f}^{2}\right\} \boldsymbol{f}^{2}\right\}$ & 126 & 1260 & 80 \\
\hline 8 & $\left\{_{2}\{(f) f\} f^{2}\right\}_{2}$ & 45 & 2520 & 448 & 8 & $\left\{\left\{f^{4}\right\} f^{2}\right\}$ & 21 & 105 & 40 \\
\hline 8 & $\left\{_{2}\left\{f^{p}\right\} f^{2}\right\}_{2}$ & 15 & 420 & 224 & 8 & $\left\{\left\{_{3} f\right\}_{3}\left\{{ }_{2} f_{2}\right\}\right.$ & 35 & 5040 & 1152 \\
\hline 8 & 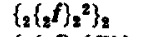 & 10 & 2520 & 2016 & 8 & $\left\{\left\{_{g} f\right\}_{g}\left\{f^{2}\right\}\right\}$ & 35 & 2520 & 576 \\
\hline 8 & $\left\{_{2}\left\{{ }_{2} f\right\}_{2}\left\{f^{2}\right\}\right\}_{2}$ & 20 & 2520 & 1008 & 8 & $\left\{\left\{_{2} \mathrm{f}^{2}\right\}_{2}\left\{{ }_{2} f\right\}_{2}\right\}$ & 35 & 2520 & 576 \\
\hline 8 & $\left\{_{2}\left\{f^{2}\right\}^{2}\right\}_{3}$ & 10 & 630 & 504 & 8 & $\left\{\left\{_{2} f^{2}\right\}_{2}\left\{f^{a}\right\}\right\}$ & 35 & 1260 & 288 \\
\hline 8 & $\left\{_{2}\left\{{ }_{2} f\right\}_{2}\{f\} f\right\}_{2}$ & 60 & 5040 & 672 & 8 & $\left\{\{\{f\} f\}\left\{_{2} f\right\}_{2}\right\}$ & 105 & 5040 & 384 \\
\hline 8 & $\left\{_{2}\left\{f^{2}\right\}\{f\} f\right\}_{2}$ & 60 & 2520 & 336 & 8 & $\left\{\{\{f\} f\}\left\{f^{2}\right\}\right\}$ & 105 & 2520 & 192 \\
\hline 8 & $\left\{\left\{_{2} f\right\}_{2} F^{\prime}\right\}_{2}$ & 20 & 840 & 336 & 8 & $\left\{\left\{f^{3}\right\}\left\{_{2} f\right\}_{2}\right\}$ & 35 & 840 & 192 \\
\hline 8 & $\left\{_{2}\left\{f^{2}\right\} f^{2}\right\}_{2}$ & 20 & 420 & 168 & 8 & $\left\{\left\{f^{3}\right\}\left\{f^{2}\right\}\right\}$ & 35 & 420 & 96 \\
\hline 8 & $\left\{_{2}\{f\}^{3}\right\}_{2}$ & 15 & 840 & 448 & 8 & $\left\{\left\{_{3} f\right\}_{8}\{f\} f\right\}$ & 105 & 5040 & 384 \\
\hline 8 & $\left\{_{3}\left\{\boldsymbol{f}^{2} \boldsymbol{f}^{2}\right\}_{2}\right.$ & 45 & 1260 & 224 & 8 & $\left\{\left\{_{2}{ }^{2}\right\}_{2}\{f\} f\right\}$ & 105 & 2520 & 192 \\
\hline 8 & $\left\{_{2}\{f\}^{4}\right\}_{2}$ & 15 & 210 & 112 & 8 & $\{\{\{f\} f\}\{\cap f\}$ & 315 & 5040 & 128 \\
\hline 8 & $\left\{f^{6}\right\}_{2}$ & I & 7 & 56 & 8 & $\left\{\left\{\boldsymbol{f}^{\boldsymbol{f}}\right\}\{\boldsymbol{f}\} \boldsymbol{f}\right\}$ & 105 & 840 & 64 \\
\hline 8 & $\left\{\left\{_{5} f\right\}_{5} f\right\}$ & 7 & 5040 & 5760 & 8 & $\left\{\left\{_{g} f_{g_{g}} f^{3}\right\}\right.$ & 35 & 840 & 192 \\
\hline 8 & $\left\{\left\{a^{2}\right\}, f\right\}$ & 7 & 2520 & 2880 & 8 & $\left\{\left\{_{2} f^{2}\right\}_{2} f^{8}\right\}$ & 35 & 420 & 96 \\
\hline 8 & $\left\{\left\{_{3}\{f\} f\right\}_{3} f\right\}$ & 21 & 5040 & 1920 & 8 & $\left\{\{\{\boldsymbol{f}\} \boldsymbol{f}\} \boldsymbol{f}^{3}\right\}$ & 105 & 840 & 64 \\
\hline 8 & $\left\{\left\{{ }_{3} f^{3}\right\}_{3} f\right\}$ & 7 & 840 & 960 & 8 & $\left\{\left\{\boldsymbol{P}^{\mathbf{p}} \boldsymbol{f}^{\mathbf{s}}\right\}\right.$ & 35 & 140 & 32 \\
\hline 8 & $\left\{\left\{\left\{_{2}\left\{z_{2} \cap\right\}_{2} \cap\right\}_{2} \cap\right\}\right.$ & 28 & 5040 & 1440 & 8 & $\left\{\left\{{ }_{2} f\right\}_{2}{ }^{2} f\right\}$ & 70 & 2520 & 288 \\
\hline 8 & $\left\{\left\{_{2}\left\{f^{2}\right)\right\}_{2} \cap\right\}$ & 28 & 2520 & 720 & 8 & $\left\{\left\{_{2} f\right\}_{2}\left\{f^{2}\right\} f\right\}$ & 140 & 2520 & 144 \\
\hline 8 & $\left\{\left\{_{g}\{f\}^{2}\right\}_{2} f\right\}$ & 21 & 2520 & 960 & 8 & $\left\{\left(f^{2}\right\}^{a} f\right\}$ & 70 & 630 & 72 \\
\hline 8 & $\left\{\left\{_{8}\{f\} f^{2}\right\}_{8} f\right\}$ & 42 & 2520 & 480 & 8 & $\left\{\{, f\}_{2}\{n\}^{2}\right\}$ & 105 & 2520 & 192 \\
\hline 8 & $\left\{\left\{, f^{4}\right\}_{g} f\right\}$ & 7 & 210 & 240 & 8 & $\left\{\left\{f^{2}\right\}\left\{f^{2}\right\}\right.$ & 105 & 1260 & 96 \\
\hline 8 & $\left\{\left\{\{, f\}_{3} f\right\} f\right\}$ & 35 & 5040 & 1152 & 8 & $\left\{\left\{_{2} f_{\mathrm{S}}\{f\} \boldsymbol{f}^{2}\right\}\right.$ & 210 & 2520 & 96 \\
\hline
\end{tabular}


TABLE 1 (continued)

\begin{tabular}{rlrrr}
\hline$r$ & $F$ & $\alpha$ & $\beta$ & $\gamma$ \\
\hline 8 & $\left\{\left\{f^{2}\right\}\{f\} f^{2}\right\}$ & 210 & 1260 & 48 \\
8 & $\left\{\left\{f_{2} f f^{4}\right\}\right.$ & 35 & 210 & 48 \\
8 & $\left\{\left\{f^{2}\right\}^{4}\right\}$ & 35 & 105 & 24 \\
8 & $\left\{\{f\}^{3} f\right\}$ & 105 & 840 & 64 \\
8 & $\left\{\{f\}^{2} f^{3}\right\}$ & 105 & 420 & 32 \\
8 & $\left\{\left\{f f^{5}\right\}\right.$ & 21 & 42 & 16 \\
8 & $\{f\}$ & 1 & 1 & 8 \\
& & & & \\
\hline
\end{tabular}

\section{The Taylor expansion for $\hat{y}$}

The derivative of $\hat{y}$ with respect to $x$ will depend on the numbers $b_{I}, a_{I J}$ $(I, J=1,2, \cdots v)$. It is convenient to define certain vector functions of these numbers and of $f(y)$ which bear a $(1-1)$ correspondence to the elementary differentials.

We define

$$
g=\sum_{I=1}^{\nu} b_{I} g^{(I)}
$$

where $\boldsymbol{g}^{(l)}$ is defined by (4) as the only weighted differential of order 1 (the degree is undefined) and generally

$$
\boldsymbol{a}=\left\langle\boldsymbol{G}_{1} \boldsymbol{G}_{2} \cdots \boldsymbol{G}_{\mathbf{s}}\right\rangle
$$

is the weighted differential corresponding to (and having the same order and degree as) $F$ in equation (8) if $G_{i}$ corresponds to $F_{i}(i=1,2, \cdots, \sigma)$.

We also write

$$
\boldsymbol{G}=\left\langle\boldsymbol{G}_{1}^{\mu_{1}} \boldsymbol{G}_{2}^{\mu_{1}} \cdots \boldsymbol{G}_{\sigma}^{\mu_{\sigma}}\right\rangle
$$

as the weighted differential corresponding to (9). The right hand side of (17) is to be defined as follows:

Suppose $G_{i}$ has components $\left(G_{i 1}, G_{i 2}, \cdots G_{i n}\right)$ where

$$
G_{i j}=\sum_{I=1}^{\nu} G_{i j}^{(n)} b_{I}
$$

then

$$
\begin{aligned}
& \left\langle G_{1} G_{2} \cdots G_{s}\right\rangle=\sum_{I=1}^{n} \sum_{J_{1}=1}^{\nu} \sum_{J_{2}=1}^{\nu} \cdots \sum_{J_{e}=1}^{\nu} \sum_{j_{1}=1}^{n} \sum_{j_{2}=1}^{n} \cdots \sum_{j_{t}=1}^{n} b_{I} a_{I J_{1}} a_{I J_{2}} \cdots \\
& a_{I J_{1}} \frac{\partial^{2} g^{(I)}}{\partial y_{s_{i}} \partial y_{i_{1}} \cdots \partial y_{s_{1}}} G_{i_{1}}^{\left(J_{j}\right)} G_{2 j_{2}}^{\left(J_{2}\right)} \cdots G_{s j_{s}}^{\left(J_{j}\right)},
\end{aligned}
$$

where the partial derivative of $\boldsymbol{g}^{(I)}$ in the right hand side indicates a differ- 
entiation of $\boldsymbol{f}(\boldsymbol{y})$ with respect to the elements of $\boldsymbol{y}$ followed by the substitution

$$
y=y_{0}+h \sum_{J=1}^{\nu} a_{I J} g^{(J)} .
$$

It is also convenient to define a set of functions of $a_{I J}, b_{r}$ alone which we will call elementary weights. These also have a $(1-1)$ correspondence to the elementary differentials. The order and degree of an elementary weight are defined as identical with the corresponding elementary differential. Each elementary weight is a homogeneous function of degree 1 in $b_{I}$ and of degree $r-1$ in $a_{I J}$ where $r$ is its order.

The elementary weight corresponding to $f$ is

$$
\phi=\sum_{I=1}^{\nu} b_{I}
$$

and the elementary weight corresponding to $\left\{F_{1} F_{2} \cdots F_{3}\right\}$ is

$$
\Phi=\left[\Phi_{1} \Phi_{2} \cdots \Phi_{s}\right] \text {, }
$$

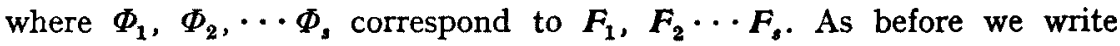

$$
\Phi=\left[\Phi_{1}^{\mu_{1}} \Phi_{2}^{\mu_{2}} \cdots \Phi_{\sigma}^{\mu_{\sigma}}\right]
$$

corresponding to (9) and we define the right hand side of (20) by the equation

$$
\left[\Phi_{1} \Phi_{2} \cdots \Phi_{s}\right]=\sum_{I=1}^{\nu} \sum_{J_{1}=1}^{\nu} \sum_{J_{2}=1}^{\nu} \cdots \sum_{J_{s}=1}^{\nu} b_{I} a_{I J_{1}} a_{I J_{2}} \cdots a_{I J_{t}} \Phi_{1}^{\left(J_{1}\right)} \Phi_{2}^{\left(J_{2}\right)} \cdots \Phi_{s}^{\left(J_{s}\right)},
$$

where

$$
\Phi_{i}=\sum_{I=1}^{\nu} b_{I} \Phi_{i}^{(I)}
$$

and $\Phi_{i}^{(I)}$ is independent of $b_{1}, b_{2}, \cdots, b_{\nu}$.

At $h=0$ the partial derivative in (19) becomes identical with that in (7) so an inductive proof to the following theorem is trivial.

ThEOREM 4. When $h=0$ and $\boldsymbol{G}, \boldsymbol{F}$ and $\Phi$ correspond

$$
\boldsymbol{a}=\Phi \boldsymbol{F} \text {. }
$$

It will be shown that the derivatives of $\hat{y}$ depend only on the weighted differentials so that the Taylor expansion for $\hat{y}$ will depend only on the elementary differentials the coefficients, however, not being constants as in the expansion for $\boldsymbol{y}$ but instead products of numerical constants and the correponding elementary weights.

Let us for example consider the first derivative of $\hat{y}$. 


$$
\frac{\mathrm{d}}{\mathrm{d} x} \boldsymbol{g}^{(I)}=\sum_{i=1}^{n} \frac{\partial g^{(I)}}{\partial y_{i}}\left(\sum_{J=1}^{\nu} a_{I J} g_{i}^{(J)}+h \sum_{j=1}^{\nu} a_{I J} \frac{\mathrm{d}}{\mathrm{d} x} g_{i}^{(J)}\right)
$$

so that

$$
\frac{\mathrm{d}}{\mathrm{d} x} \boldsymbol{g}=\langle\boldsymbol{g}\rangle+h\left\langle_{2} \boldsymbol{g}\right\rangle_{2}+h^{2}\langle\boldsymbol{g}\rangle_{3}+\cdots
$$

We note that in this example the derivative of a weighted differential of order $r$ contains terms in the weighted differentials of order greater than $r+1$.

This will be found to be generally true and in fact a straightforward differentiation of (17) gives

$$
\begin{aligned}
& \mathrm{D} G=\left\langle g G_{1} a_{2} \cdots G_{s}\right\rangle+h\left\langle\langle g\rangle G_{1} a_{2} \cdots G_{s}\right\rangle+h^{2}\left\langle\left\langle{ }_{2} g\right\rangle_{2} a_{1} G_{2} \cdots G_{s}\right\rangle \\
& +\cdots+\left\langle\left(\mathrm{D} \boldsymbol{a}_{1}\right) \boldsymbol{a}_{2} \boldsymbol{a}_{3} \cdots \boldsymbol{a}_{3}\right\rangle+\left\langle\boldsymbol{G}_{1}\left(\mathrm{D} \boldsymbol{G}_{2}\right) \boldsymbol{a}_{3} \cdots \boldsymbol{G}_{3}\right\rangle+\cdots \\
& +\left\langle G_{1} G_{2} G_{3} \cdots\left(\mathrm{D} G_{s}\right)\right\rangle \text {. }
\end{aligned}
$$

We now state the analogue of THEOREM 1.

THEOREM 5. The differential coefficient with respect to $x$ of any weighted differential of order $r$ is a power series in $h$, the coefficient of $h^{t}$ being a linear combination with non-negative integral coefficients of the weighted differentials of order $r+t+1$.

The proof is almost identical to that for Theorem 1 except that (25) plays the role played there by $(10)$.

COROLLARY 1. The sum of the coefficients of weighted differentials of order $r+t+1$ occurring in $\mathrm{DG}$ is $r h^{t}$.

COROLlaRY 2. $\mathrm{D}^{u} \boldsymbol{G}$ is a power series in $h$ the coefficient of $h^{t}$ being a linear combination with non-negative integral coefficients of weighted differentials of order $r+t+u$.

The question of the sum of the coefficients of a given order we defer to a later time.

As D $\boldsymbol{G}$ contains terms corresponding to those in the expansion of DF (and some extra ones as well) we can easily prove the analogue to Theorem 2 .

THEOREM 6. When $\mathrm{D}^{u} \boldsymbol{g}$ is written as a power series in $h$ so that the leading term is a linear combination with non-negative integral coefficients of the weighted differentials of order $1+u$, none of the coefficicnts vanishes.

It is of course this theorem which makes Runge-Kutta processes possible.

Similar to the Taylor expansion (11) we have

$$
\hat{y}=y_{0}+\sum_{i=1}^{\infty} \frac{h^{r^{(i)}}}{\left(r^{(i)}-1\right) !} \beta^{(i)} \Phi^{(i)} F^{(i)},
$$


where $F^{(1)}, F^{(2)}, \cdots, r^{(1)}, r^{(2)}, \cdots$ are as previously defined, $\Phi^{(1)}, \Phi^{(2)}, \cdots$ are the corresponding elementary weights and $\beta^{(i)}$ is the coefficient with which $\boldsymbol{G}^{(i)}$ corresponding to $\boldsymbol{F}^{(i)}$ occurs in $\mathrm{D}^{(i)} \boldsymbol{g}$ for $i=1,2, \cdots$.

Repeated application of (25) enables us to find the first few coefficients in (26). For example we find

$$
\begin{aligned}
\hat{y}= & y_{0}+h \phi \boldsymbol{f}+h^{2}[\phi]\{\boldsymbol{f}\}+h^{3}\left(\left[{ }_{2} \phi\right]_{2}\left\{{ }_{2} f\right\}_{2}+\frac{1}{2}\left[\phi^{2}\right]\left\{\boldsymbol{f}^{2}\right\}\right) \\
& +h^{\mathbf{4}}\left(\left[{ }_{3} \phi\right]_{3}\left\{_{3} f\right\}_{3}+[\phi[\phi]]\{\boldsymbol{f}\{\boldsymbol{f}\}\}+\frac{1}{2}\left[{ }_{2} \phi^{2}\right]_{2}\left\{f_{2} f^{2}\right\}_{2}+\frac{1}{6}\left[\phi^{3}\right]\left\{\boldsymbol{f}^{3}\right\}\right)+\cdots
\end{aligned}
$$

so that the specification for a fourth order Runge-Kutta process would be

$$
\begin{aligned}
\phi & =1, & {[\phi] } & =\frac{1}{2}, \\
\left.{ }_{2} \phi\right]_{2} & =\frac{1}{6}, & {\left[\phi^{2}\right] } & =\frac{1}{3}, \\
{\left[{ }_{3} \phi\right]_{3} } & =\frac{1}{24}, & {[\phi[\phi]] } & =\frac{1}{8}, \\
\left.{ }_{2} \phi^{2}\right]_{2} & =\frac{1}{12}, & {\left[\phi^{3}\right] } & =\frac{1}{4},
\end{aligned}
$$

or in expanded form for $v=4$ and with the restriction (6)

$$
\begin{aligned}
b_{1}+b_{2}+b_{3}+b_{4} & =1, & b_{2} c_{2}+b_{3} c_{3}+b_{4} c_{4} & =\frac{1}{2}, \\
b_{3} a_{32} c_{2}+b_{4}\left(a_{42} c_{2}+a_{43} c_{3}\right) & =\frac{1}{6}, & b_{2} c_{2}^{2}+b_{3} c_{3}^{2}+b_{4} c_{4}^{2} & =\frac{1}{3}, \\
b_{4} a_{43} a_{32} c_{2} & =\frac{1}{24}, & b_{3} c_{3} a_{32} c_{2}+b_{4} c_{4}\left(a_{42} c_{2}+a_{43} c_{3}\right) & =\frac{1}{8}, \\
b_{3} a_{32} c_{2}^{2}+b_{4}\left(a_{42} c_{2}^{2}+a_{43} c_{3}^{2}\right) & =\frac{1}{12}, & b_{2} c_{2}^{3}+b_{3} c_{3}^{3}+b_{4} c_{4}^{3} & =\frac{1}{4},
\end{aligned}
$$

where

$$
c_{2}=a_{21}, \quad c_{3}=a_{31}+a_{32}, \quad c_{4}=a_{41}+a_{42}+a_{43} .
$$

In general if the Runge-Kutta process is to be accurate up to terms in $h^{r}$ (11) and (26) must agree to this accuracy so that

$$
\Phi^{(i)}=\frac{1}{\gamma^{(i)}} \cdot \frac{\alpha^{(i)}}{\beta^{(i)}}=\frac{1}{\gamma^{(i)}},
$$

say, for all $i$ such that $r^{(i)} \leqq r$.

The use of (25) to find the values of $\beta^{(i)}$ is even more tedious than is the use of $(10)$ to find $\alpha^{(i)}$. However, we have corresponding to Theorem 3 the following result.

THEOREM 7. If $\left(h^{r} /(r-1)\right.$ !) $\beta \Phi$ is the coefficient of $F$ defined by (9) in the expansion (26) where $\Phi$ is the corresponding elementary weight and $F_{1}, F_{2}, \cdots$ $F_{\sigma}$ are all distinct then

$$
\beta=(r-1) ! \prod_{i=1}^{\sigma} \frac{1}{\mu_{i} !}\left(\frac{\beta_{i}}{\left(r_{i}-1\right) !}\right)^{\mu_{i}},
$$

where $\left(h^{r} /\left(r_{i}-1\right)\right.$ !) $\beta_{i} \Phi_{i}(i=1,2, \cdots, \sigma)$ is the coefficient of $F_{i}$ in (26) and $\Phi_{i}$ corresponds to $F_{i}$. 
To prove this result we confine ourselves to the special case $v=1$, $b_{1}=a_{11}=1$. This restriction is unimportant as the terms in (25) do not depend on these parameters. (5) now becomes

$$
\dot{y}=y_{0}+h f(\hat{y}) \text {. }
$$

Substitute (26) into this equation with $\Phi^{(i)}$ now set equal to unity and we obtain a coefficient for $\boldsymbol{F}$ on the left hand side

$$
\frac{\beta h^{r}}{(r-1) !}
$$

while the right hand side can be formally written as

where

$$
\hat{\eta}=\sum_{i=1}^{\infty} \frac{h^{(i)}}{\left(r^{(i)}-1\right) !} \beta^{(i)} F^{(i)}
$$

Thus the terms in (30) are identical to those in (14) except that $\alpha_{i}$ is to be replaced by $r_{i} \beta_{i}$ and an extra factor $h$ is present. Thus the coefficient of $F$ in $(30)$ is

$$
\begin{aligned}
\frac{h^{r}}{s !}\left(\begin{array}{c}
s \\
\mu_{1}, \mu_{2}, \cdots
\end{array}\right) \prod_{\sigma}^{\sigma}\left(\frac{\beta_{i}}{\left(r_{i}-1\right) !}\right)^{\mu_{i}} \\
=h^{r} \prod_{i=1}^{\sigma} \frac{1}{\mu_{i} !}\left(\frac{\beta_{i}}{\left(\gamma_{i}-1\right) !}\right)^{\mu_{i}}
\end{aligned}
$$

and a comparison of this with (29) gives the result (28).

The fourth column in Table 1 gives the corresponding values of $\beta$ to the elementary differentials in the second column. As with the values of $\alpha$, the sum of all values of $\beta$ for a given order has a simple expression. Let this sum for order $r$ be $B_{r}$ and consider the function of $\xi$ say

$$
B=B_{1} \xi+\frac{1}{1 !} B_{2} \xi^{2}+\frac{1}{2 !} B_{3} \xi^{3}+\cdots
$$

A comparison of $(29)$ and $(30)$ gives us that

$$
\begin{aligned}
B & =\xi\left(1+B+\frac{1}{2 !} B^{2}+\cdots\right) \\
& =\xi e^{B} .
\end{aligned}
$$

The solution to this equation corresponding to $B=0$ at $\xi=0$ is [6],

$$
B_{r}=r^{r-2} \text {. }
$$


The values of $\gamma$ defined in (27) are useful and these can be found from the relation

$$
\gamma=\frac{r \beta}{\alpha}
$$

However, by comparing (28) with (12) we find an inductive means of computing this quantity independently. We find

$$
\gamma=r \prod_{i=1}^{\sigma} \gamma_{i}^{\boldsymbol{*}^{\prime}}
$$

where $\gamma_{i}$ corresponds to $\alpha_{i}, \beta_{i}$.

\section{A process of order 5}

As an example of the preceding theory we shall derive the parameters for a 3 stage process of 5 th order accuracy.

We define

$$
\begin{aligned}
& c_{1}=a_{11}+a_{12}+a_{13}, \\
& c_{2}=a_{21}+a_{22}+a_{23}, \\
& c_{3}=a_{31}+a_{32}+a_{33},
\end{aligned}
$$

and suppose that $b_{1}, b_{2}, b_{3}, c_{1}, c_{2}, c_{3}$ are chosen so that

$$
\begin{aligned}
\phi & \equiv b_{1}+b_{2}+b_{3}=1, \\
{[\phi] } & \equiv b_{1} c_{1}+b_{2} c_{2}+b_{3} c_{3}=\frac{1}{2}, \\
{\left[\phi^{2}\right] } & \equiv b_{1} c_{1}^{2}+b_{2} c_{2}^{2}+b_{3} c_{3}^{2}=\frac{1}{3}, \\
{\left[\phi^{3}\right] } & \equiv b_{1} c_{1}^{3}+b_{2} c_{2}^{3}+b_{3} c_{3}^{3}=\frac{1}{4}, \\
{\left[\phi^{4}\right] } & \equiv b_{1} c_{1}^{4}+b_{2} c_{2}^{4}+b_{3} c_{3}^{4}=\frac{1}{5} .
\end{aligned}
$$

It is clear that none of $b_{1}, b_{2}, b_{3}$ can vanish and that $c_{1}, c_{2}, c_{3}$ are distinct.

The equations $\left[{ }_{2} \phi\right]_{2}=\frac{1}{6},[[\phi] \phi]=\frac{1}{8},\left[[\phi] \phi^{2}\right]=\frac{1}{10}$ when combined with equations (35), (36), (37) respectively give

$$
\begin{gathered}
b_{1}\left(\sum_{I=1}^{3} a_{1 I} c_{I}-\frac{1}{2} c_{1}^{2}\right)+b_{2}\left(\sum_{I=1}^{3} a_{2 I} c_{I}-\frac{1}{2} c_{2}^{2}\right)+b_{3}\left(\sum_{I=1}^{3} a_{3 I} c_{I}-\frac{1}{2} c_{3}^{2}\right)=0, \\
b_{1} c_{1}\left(\sum_{I=1}^{3} a_{I I} c_{I}-\frac{1}{2} c_{1}^{2}\right)+b_{2} c_{2}\left(\sum_{I=1}^{3} a_{2 I} c_{I}-\frac{1}{2} c_{2}^{2}\right)+b_{3} c_{3}\left(\sum_{I=1}^{3} a_{3 I} c_{I}-\frac{1}{2} c_{3}^{2}\right)=0, \\
b_{1} c_{1}^{2}\left(\sum_{I=1}^{3} a_{I I} c_{I}-\frac{1}{2} c_{1}^{2}\right)+b_{2} c_{2}^{2}\left(\sum_{I=1}^{3} a_{2 I} c_{I}-\frac{1}{2} c_{2}^{2}\right)+b_{3} c_{3}^{2}\left(\sum_{I=1}^{3} a_{3 I} c_{I}-\frac{1}{2} c_{3}^{2}\right)=0 .
\end{gathered}
$$

Hence, since the matrix 


$$
\left[\begin{array}{lll}
b_{1} & b_{2} & b_{3} \\
b_{1} c_{1} & b_{2} c_{2} & b_{3} c_{3} \\
b_{1} c_{1}^{2} & b_{2} c_{2}^{2} & b_{3} c_{3}^{2}
\end{array}\right]
$$

has non vanishing determinant $b_{1} b_{2} b_{3}\left(c_{1}-c_{2}\right)\left(c_{2}-c_{3}\right)\left(c_{3}-c_{1}\right)$ we have

$$
\begin{aligned}
& a_{11} c_{1}+a_{12} c_{2}+a_{13} c_{3}=\frac{1}{2} c_{1}^{2}, \\
& a_{21} c_{1}+a_{22} c_{2}+a_{23} c_{3}=\frac{1}{2} c_{2}^{2}, \\
& a_{31} c_{1}+a_{32} c_{2}+a_{33} c_{3}=\frac{1}{2} c_{3}^{2} .
\end{aligned}
$$

Similarly, the equations $\left[{ }_{2} \phi^{2}\right]_{2}=\frac{1}{12},\left[\left[\phi^{2}\right] \phi\right]=\frac{1}{16}$ will be satisfied if

$$
\begin{aligned}
& a_{11} c_{1}^{2}+a_{12} c_{2}^{2}+a_{13} c_{3}^{2}=\frac{1}{3} c_{1}^{3}, \\
& a_{21} c_{1}^{2}+a_{22} c_{2}^{2}+a_{23} c_{3}^{2}=\frac{1}{3} c_{2}^{3}, \\
& a_{31} c_{1}^{2}+a_{32} c_{2}^{2}+a_{33} c_{3}^{2}=\frac{1}{3} c_{3}^{3},
\end{aligned}
$$

and it is convenient to assume these conditions which, together with (32) and (38), may be used to find $a_{I J}(I, J=1,2,3)$ once the values of $c_{1}, c_{2}, c_{3}$ are chosen.

The equation $\left.{ }_{3} \phi\right]_{3}=\frac{1}{24}$ is $\sum_{I, J, K=1}^{3} b_{I} a_{I J} a_{J K} c_{K}=\frac{1}{24}$ and the left-hand side is

$$
\sum_{I, J=1}^{3} b_{I} a_{I J}\left(\frac{1}{2} c_{J}^{2}\right)=\frac{1}{6} \sum_{I=1}^{3} b_{I} c_{I}^{3}=\frac{1}{24},
$$

so that all elementary weights of order less than 5 have now been seen to have the correct value.

It is now easy to verify that $\left[\left[{ }_{2} \phi\right]_{2} \phi\right]=\frac{1}{30}$ and $\left[[\phi]^{2}\right]=\frac{1}{20}$. We have and

$$
\left[\left[{ }_{2} \phi\right]_{2} \phi\right]=\sum_{I, J, K=1}^{3} b_{I} c_{I} a_{I J} a_{J K} c_{K}=\frac{1}{2} \sum_{I, J=1}^{3} b_{I} c_{I} a_{I J} c_{J}^{2}=\frac{1}{6} \sum_{I=1}^{3} b_{I} c_{I}^{4}=\frac{1}{30}
$$

$$
\left[[\phi]^{2}\right]=\sum_{I=1}^{3} b_{I}\left(\sum_{J=1}^{3} a_{I J} c_{J}\right)^{2}=\frac{1}{4} \sum_{I=1}^{3} b_{I} c_{I}^{4}=\frac{1}{20}
$$

It now remains to prove $\Phi=1 / \gamma$ when $\Phi$ is of order 5 and degree 1 . However, first we will prove that, assuming $b_{1}, b_{2}, b_{3}, a_{11}, a_{12}, \cdots, a_{33}$ to be chosen to satisfy equations $(32)-(39)$, then

$$
\begin{aligned}
& b_{1} a_{11}+b_{2} a_{21}+b_{3} a_{31}=b_{1}\left(1-c_{1}\right), \\
& b_{1} a_{12}+b_{2} a_{22}+b_{3} a_{32}=b_{2}\left(1-c_{2}\right), \\
& b_{1} a_{13}+b_{2} a_{23}+b_{3} a_{33}=b_{3}\left(1-c_{3}\right) .
\end{aligned}
$$

This will be true if 


$$
\begin{gathered}
u\left(b_{1} a_{11}+b_{2} a_{21}+b_{3} a_{31}-b_{1}\left(1-c_{1}\right)\right)+v\left(b_{1} a_{12}+b_{2} a_{22}+b_{3} a_{32}-b_{2}\left(1-c_{2}\right)\right) \\
+w\left(b_{1} a_{13}+b_{2} a_{23}+b_{3} a_{33}-b_{3}\left(1-c_{3}\right)\right)=0
\end{gathered}
$$

for three independent vectors $(u, v, w)$. This is easily verified for the three vectors $(1,1,1),\left(c_{1}, c_{2}, c_{3}\right),\left(c_{1}^{2}, c_{2}^{2}, c_{3}^{2}\right)$ so the result follows.

Now consider an elementary weight of degree 1 and order 5 ,

where

$$
\Phi=\left[\Phi^{\prime}\right]
$$

$$
\Phi^{\prime}=\left[\Phi_{1} \Phi_{2} \cdots \Phi_{s}\right]
$$

is of degree $s$ and order 4 .

We have $\Phi_{1}=1 / \gamma_{1}, \Phi_{2}=1 / \gamma_{2}, \cdots, \Phi_{s}=1 / \gamma_{s}, \Phi^{\prime}=1 / \gamma^{\prime}$ where $\gamma^{\prime}=$ $4 \gamma_{1} \gamma_{2} \cdots \gamma_{s}$ (from (31)) and we wish to prove $\Phi=1 / \gamma$ where $\gamma=5 \gamma^{\prime}$.

Let us now define $\Phi^{\prime \prime}=\left[\phi \Phi_{1} \Phi_{2} \cdots \Phi_{s}\right]$, of order 5 and degree greater than 1, so that $\Phi^{\prime \prime}=1 / \gamma^{\prime \prime}$ where $\gamma^{\prime \prime}=5 \gamma_{1} \gamma_{2} \cdots \gamma_{s}=\frac{1}{4} \gamma$. Using (22), (40) we find

the correct value.

$$
\begin{aligned}
\Phi & =\Phi^{\prime}-\Phi^{\prime \prime} \\
& =\frac{1}{\gamma^{\prime}}-\frac{1}{\gamma^{\prime \prime}}=\frac{1}{\gamma},
\end{aligned}
$$

It now remains to choose a convenient solution to $(33)-(37)$ and to find the values of $a_{11}, a_{12}, \cdots a_{33}$ from (32), (38), (39). For simplicity we suppose $c_{1}=0$ so that $a_{11}=a_{12}=a_{13}=0$.

We now find

$$
\begin{array}{llll}
c_{2}=\frac{6-\sqrt{ } 6}{10}, & c_{3}=\frac{6+\sqrt{ } 6}{10}, & b_{1}=\frac{1}{9}, & b_{2}=\frac{16+\sqrt{ } 6}{36}, \\
b_{3}=\frac{16-\sqrt{ } 6}{36}, & a_{21}=\frac{9+\sqrt{ } 6}{75}, & a_{22}=\frac{24+\sqrt{ } 6}{120}, & a_{23}=\frac{168-73 \sqrt{ } 6}{600}, \\
a_{31}=\frac{9-\sqrt{ } 6}{75}, & a_{32}=\frac{168+73 \sqrt{ } 6}{600}, & a_{33}=\frac{24-\sqrt{ } 6}{120} .
\end{array}
$$

Thus we have for a possible process in which $g^{(1)}$ is defined explicitly and $\boldsymbol{g}^{(2)}, \boldsymbol{g}^{(3)}$ implicitly,

$$
\begin{aligned}
g^{(1)} & =f\left(y_{0}\right), \\
g^{(2)} & =f\left(y_{0}+h\left(\frac{9+\sqrt{ } 6}{75} g^{(1)}+\frac{24+\sqrt{ } 6}{120} g^{(2)}+\frac{168-73 \sqrt{ } 6}{600} g^{(3)}\right)\right) \\
g^{(3)} & =f\left(y_{0}+h\left(\frac{9-\sqrt{ } 6}{75} g^{(1)}+\frac{168+73 \sqrt{ } 6}{600} g^{(2)}+\frac{24-\sqrt{ } 6}{120} g^{(3)}\right)\right) \\
\hat{y} & =y_{0}+h\left(\frac{1}{9} g^{(1)}+\frac{16+\sqrt{ } 6}{36} g^{(2)}+\frac{16-\sqrt{ } 6}{36} g^{(3)}\right) \\
& =y+O\left(h^{6}\right) .
\end{aligned}
$$


It is remarkable that the choice of 12 independent parameters $a_{11}, a_{12}, \cdots$, $a_{33}, b_{1}, b_{2}, b_{3}$ has enabled us to satisfy no less than 17 separate equations. It happens that this situation is capable of extensive generalization and, for example, keeping this same value $\nu=3$ it is possible to satisfy the 37 conditions necessary for a sixth order process. Similarly for any value of $v$ a process of order up to $2 v$ is possible. It is intended that details of such processes will be discussed in a later publication.

\section{References}

[1] Runge, C., Utber die numerische Auflösung von Differentialgleichungen. Math. Ann. 46 (1895), $187-178$.

[2] Kutta, W., Beitrag zur näherungsweisen Integration totaler Differentialgleichungen. Zeit. Math. Physik, 46 (1901), 435-452.

[3] Nyström, E. J., Uber die numerische Integration von Differentialgleichungen. Acta Soc. Sci. Fennicae 50, No. 13 (1925).

[4] Gill, S., A process for the step-by-step Integration of Differential Equations in an Automatic Digital Computing Machine. Proc. Camb. Phil. Soc. 47 (1951), 96-108.

[5] Merson, R. H., An operational method for the study of integration processes. Proceedings of conference on data processing and Automatic Computing Machines at Weapons Research Establishment, Salisbury, South Australia (1957).

[6] Polya, G. and Szegö, G., Aufgaben und Lehrsatze aus der Analysis I, p. 301. Grund. Math. Wiss. 19 (Springer, 1925).

Department of Mathematics,

University of Canterbury,

Christchurch, New Zealand. 